\title{
Mechanism of action of flibanserin, a multifunctional serotonin agonist and antagonist (MSAA), in hypoactive sexual desire disorder
}

\author{
Stephen M. Stahl \\ ISSUE: \\ Flibanserin is a novel multifunctional serotonin agonist and antagonist (MSAA) that \\ improves sexual functioning in premenopausal women who suffer from reduced \\ sexual interest and desire.
}

\section{Take-Home Points}

- Sexual desire is regulated not only by the sex hormones testosterone and estrogen, but also by the neurotransmitters dopamine and norepinephrine, which enhance sexual interest and desire, and serotonin, which inhibits sexual interest and desire.

- Brain circuits that connect the prefrontal cortex (PFC) with limbic pleasure centers theoretically mediate motivation, interest, and desire. These circuits are hypothesized to be the sites of inefficient information processing associated with sexual disorders that are characterized by reduced interest and desire.

- The multifunctional serotonergic agent flibanserin is both a serotonin $1 \mathrm{~A}$ agonist and a serotonin $2 \mathrm{~A}$ antagonist. Flibanserin theoretically improves sexual functioning by enhancing downstream release of dopamine and norepinephrine while reducing serotonin release in the brain circuits that mediate symptoms of reduced sexual interest and desire.

\section{Introduction}

Brain circuits of motivation and pleasure include frontostriatal pathways and neuronal projections involving the insula, amygdala, hypothalamus, and ventral striatum. ${ }^{1-4}$ These brain areas hypothetically process rewarding stimuli, including sex, food, and drugs of abuse, along with other pleasurable stimuli, including the "runner's high" of jogging, certain social experiences, relationships, achievements, aesthetics, and intellectual pursuits. ${ }^{1}$ Recent research suggests that these same brain circuits are also involved when patients experience a lack of interest in and desire for sex. ${ }^{5,6}$ Studies also indicate that a new therapeutic agent, flibanserin, improves interest in and desire for sex by hypothetically targeting these circuits and causing the release of dopamine and norepinephrine while also reducing the release of serotonin. ${ }^{7-11}$

\section{Reward Processing as a Symptom Dimension in Psychiatric Disorders}

Various psychiatric disorders affect reward processing and are associated with the loss of interest and desire to pursue pleasurable activities because they are no longer engaging or rewarding. Reward processing is one of the key symptom domains in the modern "dimensional approach" to psychiatric disorders ${ }^{12,13}$ because abnormal reward processing hypothetically causes symptoms that can cut across a wide number of conditions, including major depressive disorder (MDD), schizophrenia, dementia, substance abuse, eating disorders, and sexual disorders. ${ }^{14}$ Symptoms may include generalized anhedonia; reduced positive affect; and lack of energy, enthusiasm, happiness, and selfconfidence. ${ }^{1,12,13}$ Reward processing is sometimes very specific, and various disorders can selectively disrupt 
interest in and desire for a particular type of reward. One example of this is a condition of reduced interest in and desire for sexual activity, called variously hypoactive sexual desire disorder (HSDD) or female sexual interest and arousal disorder (FSIAD) ${ }^{15}$ Regardless of the disorder's label, ${ }^{15,16}$ if a woman suffers from prolonged loss of sexual desire that causes her distress and cannot be explained by problematic relationships, stressors, or medical conditions, then it is considered to be a disorder of reward processing for sex, and as such is hypothetically mediated by inefficient information processing in reward circuits. ${ }^{1-6}$

\section{Disorder of Sexual Interest and Desire, or of Arousal?}

Interest in and desire for sexual activity is the first phase of the human sexual response, followed by arousal, and then by orgasm (accompanied in men by ejaculation). ${ }^{1,4,17,18}$ Disorders of orgasm and ejaculation have been recognized ever since the introduction of Prozac (fluoxetine) and the other selective serotonin reuptake inhibitors (SSRIs), which raise serotonin and commonly inhibit or delay orgasm/ejaculation. ${ }^{18}$ Disorders of arousal became well known with the introduction of Viagra (sildenafil citrate) and other nitric oxide synthase (NOS) inhibitors, which raise nitric oxide levels and enhance arousal, improving erections in men. ${ }^{18}$ Finally, disorders of interest in and desire for sex have also long been identified as a consequence of agents that enhance the release of serotonin (eg, SSRIs) or that block the release of dopamine (antipsychotics). ${ }^{3,4,8,18}$

However, until recently, primary disorders of interest in and desire for sex have not been well recognized. ${ }^{1-6}$ Recent changes in diagnostic criteria for sexual disorders have arguably made it even more difficult to reach a primary diagnosis of loss of interest in and desire for sex. ${ }^{15}$ Confusion exists because some diagnostic systems now merge disorders of interest and desire in women with disorders of arousal, while other diagnostic systems keep them separate. ${ }^{15,16}$ Fortunately, as often occurs when a new therapeutic emerges, things are becoming clearer. It now seems that the primary disorder of loss of sexual interest and desire (not a disorder of arousal or orgasm) can be recognized and treated successfully when it occurs in premenopausal women. ${ }^{7,18-23}$

\section{Microcircuits and Sexual Interest and Desire}

A microcircuit is one way to describe what happens at the level of a synapse between 2 neurons (see boxes in
Figures 1-3). This is classically where neurotransmitters regulate brain functions and where drugs act. For example, each phase of the human sexual response appears to be regulated by different neurotransmitters operating within different microcircuits. ${ }^{1,4,17,18}$ Interest and desire-sometimes called libido-are positively regulated not only by synaptic (microcircuit) dopamine (Figure 1A), but also by other microcircuits where norepinephrine (Figure 2A), testosterone, and estrogen act. Libido is also negatively modulated by microcircuits where prolactin and serotonin act (Figure 3A). ${ }^{1,4,17}$ Disorders of sexual interest and desire are hypothetically linked therefore to relative deficiencies in microcircuit dopamine (compare the microcircuits in Figures 1A and 1B) and norepinephrine (compare the microcircuits in Figures 2A and 2B) and a relative excess in microcircuit serotonin (compare the microcircuits in Figures $3 \mathrm{~A}$ and $3 \mathrm{~B}){ }^{1,4,17}$ Furthermore, prefrontal cortex (PFC) circuits utilizing glutamate may be overly active when sexual desire is low. ${ }^{2}$ HSDD may therefore be considered a maladaptation of the brain that arises from excessive excitatory signaling from the PFC to subcortical reward-related structures, which in turn leads to aberrant processing of information from rewarding events in both limbic and cortical areas.

Neurotransmitters acting in still other microcircuits (not shown) regulate sexual arousal, which is positively affected by nitric oxide and acetylcholine (and negatively by anticholinergics). ${ }^{1,4,17,18}$ Finally, orgasm is regulated in microcircuits (not shown) positively affected by norepinephrine (and negatively by serotonin). ${ }^{1,4,17,18}$ The pharmacologic mechanisms of drugs are similarly described by their sites of action in microcircuits. Thus, SSRIs work on presynaptic serotonin transporters to raise synaptic serotonin levels; NOS inhibitors block the enzyme NOS and raise nitric oxide levels in the cytoplasm of pre- and post-synaptic neurons at the microcircuits where they synapse. ${ }^{17,18}$

Flibanserin has demonstrated clinical efficacy in premenopausal women who have reduced interest in and desire for sex ${ }^{19-24}$ and has 2 principal pharmacologic actions in microcircuits: it is a full agonist at postsynaptic serotonin $5 \mathrm{HT} 1 \mathrm{~A}$ receptors and an antagonist at postsynaptic 5HT2A receptors (Figure 4). ${ }^{7-11}$ How do these actions of flibanserin reduce sexual dysfunction and enhance sexual interest and desire? The answer may lie in how flibanserin works in macrocircuits, altering downstream neurotransmitter release and improving the efficiency of information processing in those specific neuronal networks that regulate sexual desire. 


\section{BRAINSTORMS—Clinical Neuroscience Update}

Figure 1. Diagrams depicting proposed actions of flibanserin on dopamine (DA) centers of the brain. The figure depicts the dopaminergic system (A) before and (B) after administration of flibanserin. Reciprocal innervation between the brainstem and the prefrontal cortex (PFC) explains why glutamate (glu) can regulate dopamine, and vice versa.

A

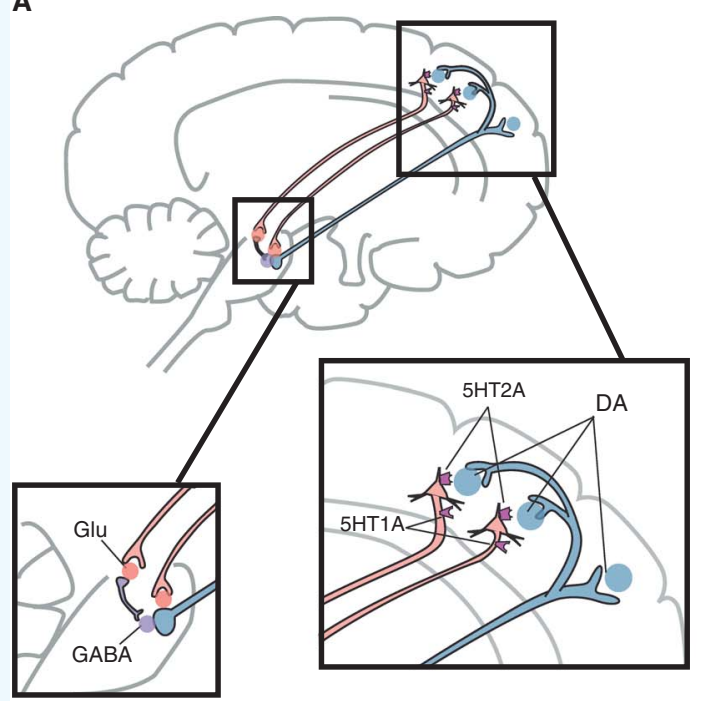

B

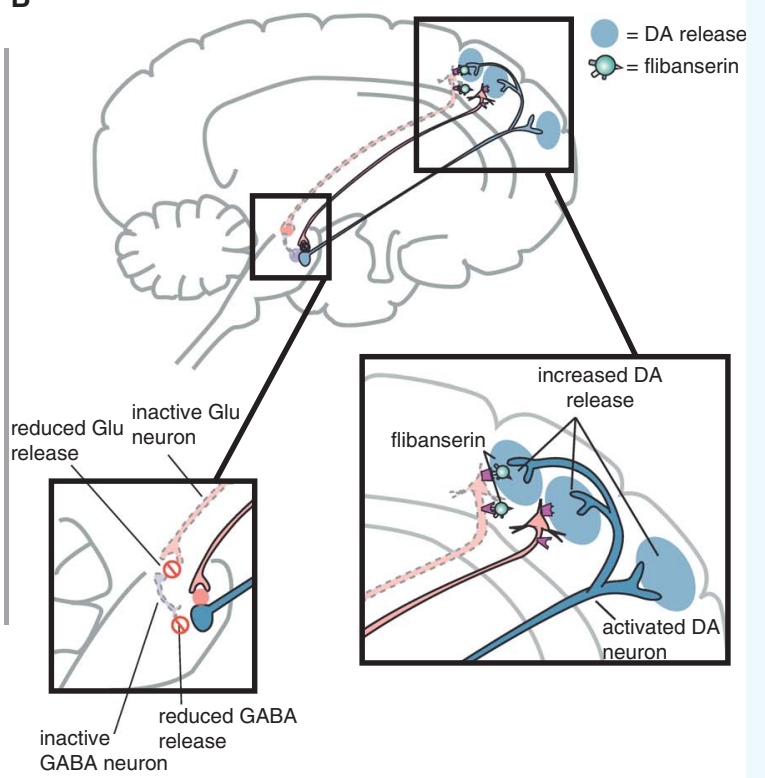

Figure 2. Diagrams depicting proposed actions of flibanserin on norepinephrine (NE) centers of the brain. The figure depicts the noradrenergic system (A) before and (B) after administration of flibanserin. Reciprocal innervation between the brainstem and the prefrontal cortex (PFC) explains why glutamate (glu) can regulate norepinephrine, and vice versa.

A

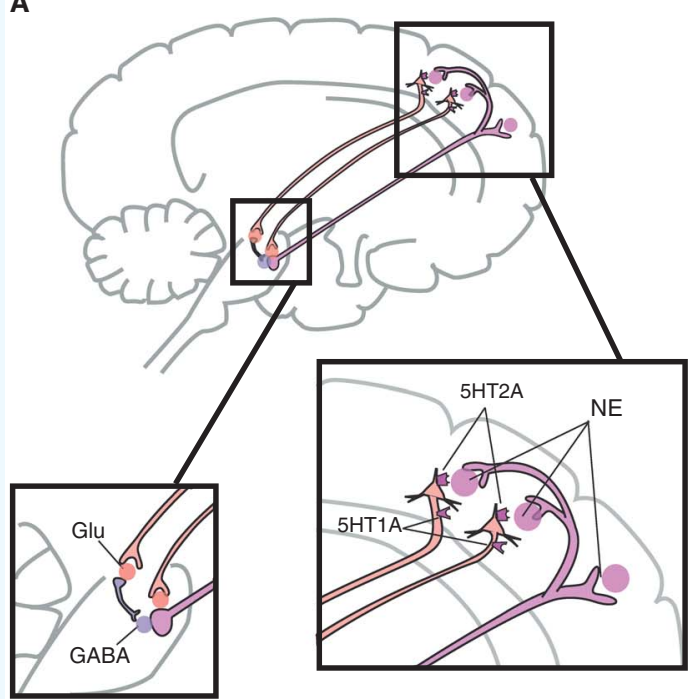

B

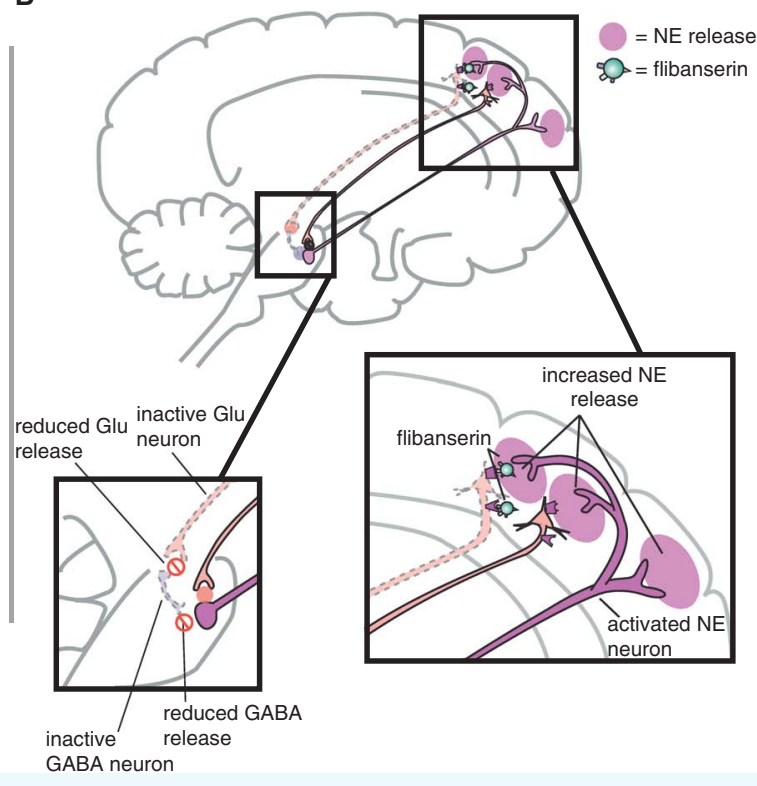




\section{BRAINSTORMS-Clinical Neuroscience Update}

Figure 3. Diagrams depicting proposed actions of flibanserin on serotonin $(5 \mathrm{HT})$ centers of the brain. The figure depicts the serotonergic system (A) before and (B) after administration of flibanserin. Reciprocal innervation between the brainstem and the prefrontal cortex (PFC) explains why glutamate (glu) can regulate serotonin, and vice versa.

A

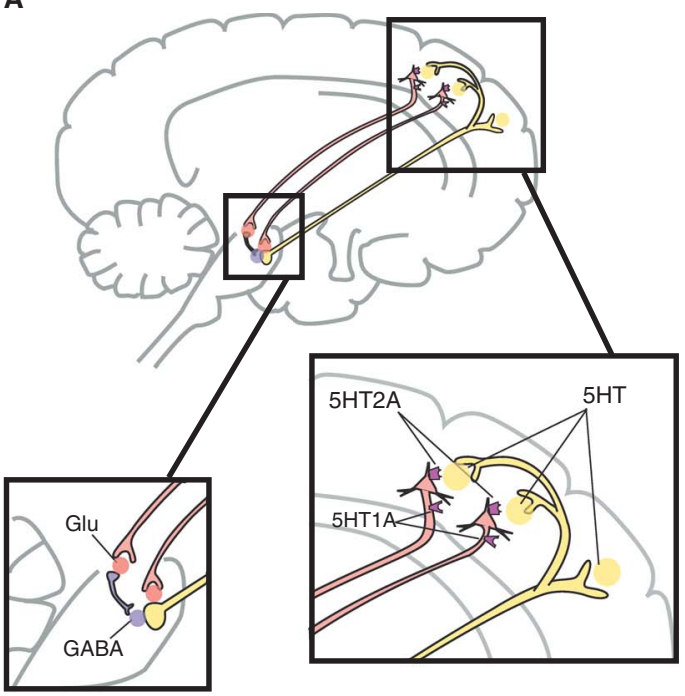

B

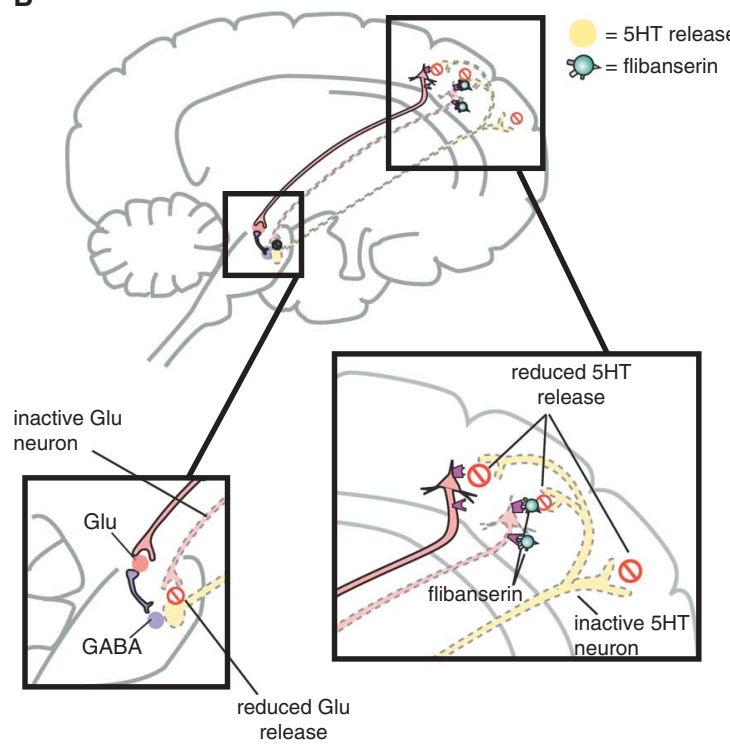

Figure 4. Top: Icon representing flibanserin's pharmacologic actions. Flibanserin's primary actions are as a 5HT1A agonist and 5HT2A antagonist. Bottom: Schematic of flibanserin binding at different human neurotransmitter receptors (nM).

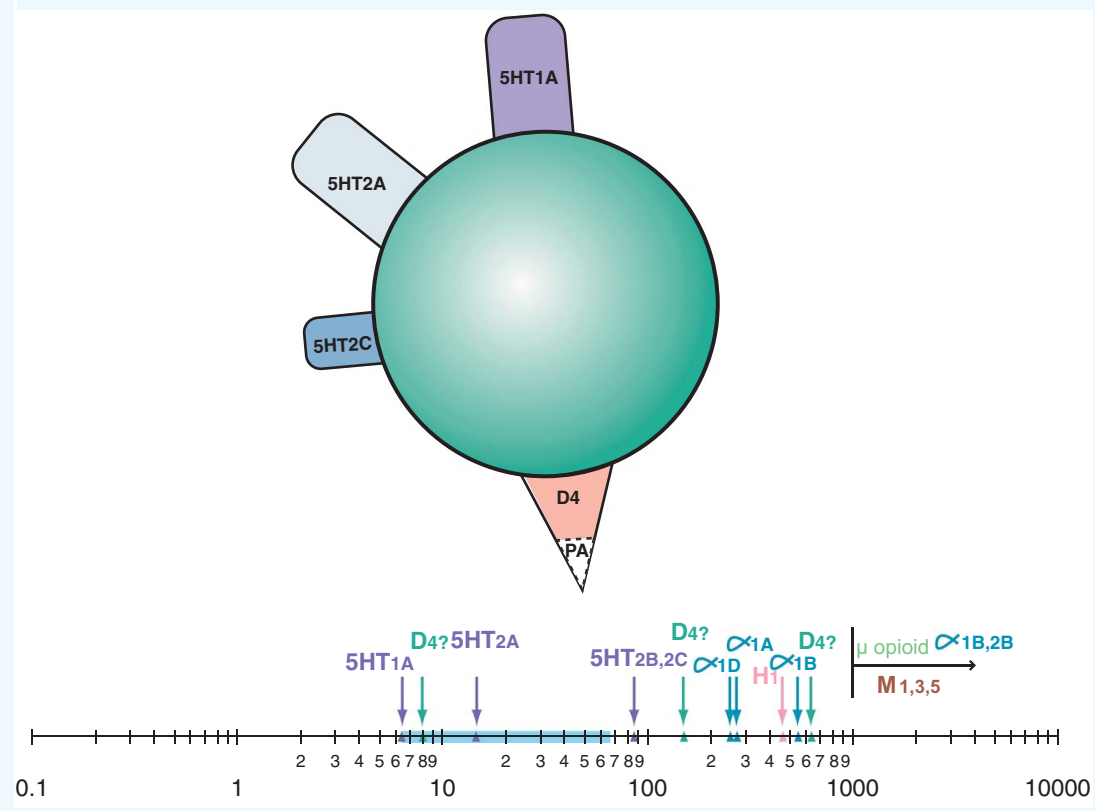




\section{Macrocircuits and Sexual Interest and Desire}

Modern neuroscience is moving beyond describing psychiatric disorders, neurotransmitter actions, and drug mechanisms simply at the level of microcircuits. With modern neuroimaging methods, the activity of specific brain areas can be visualized in living human subjects and used to describe disease actions and drug actions within a network of connected neurons, sometimes called macrocircuits. ${ }^{1-4,12,13}$ Although much further research is required to clarify and replicate these findings, neuroimaging studies in women with a disorder of sexual interest and desire already implicate differences in brain activity in macrocircuits known to regulate reward., ${ }^{5,6}$ For example, functional magnetic resonance imaging has revealed abnormal activation in cortical and striatal regions in women with HSDD. ${ }^{6}$ ) It is unknown whether these changes are due to unwanted activation of inhibitory networks that suppress sexual interest and desire, presumably at the prefrontal cortical level, or to the inability to activate reward processing to acquire, encode, and retrieve reward-related memories at the striatal and limbic levels., ${ }^{5,6}$ However, these findings implicate potential sites of hypothetical macrocircuit malfunctioning in those women who experience a disorder of sexual interest and desire.

Contemporary psychiatric research now takes the approach of deconstructing the symptoms of any disorder into its component symptoms, and then mapping these symptoms onto hypothetically malfunctioning macrocircuits. The brain is topographically organized in such a way that different functions are regulated in different brain areas and, thus, different symptoms are thought to arise from inefficient information processing in different brain areas. ${ }^{12,13}$ The idea for treatment is to target these specific macrocircuits with agents that change neurotransmitter action at these sites, improve the efficiency of information processing, and reduce symptoms. For premenopausal women with reduced interest in and desire for sex, this means mapping this symptom domain to frontostriatal and limbic brain macrocircuits shown in neuroimaging studies ${ }^{5,6}$ and targeting these macrocircuits with therapeutic agents that increase dopamine, increase norepinephrine, decrease serotonin, or perform some combination of these actions. ${ }^{1,4,17,18}$

\section{Flibanserin as a Multifunctional Serotonin Agonist and Antagonist (MSAA): Modulator of Reward in Macrocircuits}

It is not easy to measure neurotransmitter release in specific brain areas of living humans, and is not even feasible for most neurotransmitters in most brain areas. However, it is possible to model what regional effects a drug has in living animals utilizing microdialysis techniques. Furthermore, changes in neurotransmitter levels can be measured at various nodes within brain networks-in other words, within specific brain regions of various macrocircuits. ${ }^{7-11,25}$ Research of this nature that has been performed with flibanserin indicates that this agent enhances the release of both dopamine (Figure 1B) and norepinephrine (Figure 2B) and reduces the release of serotonin (Figure $3 \mathrm{~B}$ ) in prefrontal brain areas consistent with the same sites of abnormal neuroimaging described in patients with reduced sexual interest and desire. 5,6 Thus, one can describe the hypothetical mechanism of action of flibanserin as stimulating postsynaptic 5HT1A receptors and blocking postsynaptic 5HT2A receptors in the prefrontal cortex, causing the downstream release of dopamine and norepinephrine and the reduction of serotonin in those brain circuits, thus regulating reward processing in premenopausal women with reduced sexual interest and desire. ${ }^{7}$

Interestingly, preclinical and functional evidence has revealed that flibanserin shows functional preference for 5HT1A and 5HT2A receptors in the cortex, over other brain regions. Furthermore, as shown in Figures 1-3, flibanserin appears to distinguish some postsynaptic 5HT1A and 5HT2A receptors from others in the PFC. Flibanserin's actions are consistent with the possibility that it acts selectively on pyramidal neurons that excite brainstem 5HT neurons, yet also selectively on pyramidal neurons that inhibit brainstem NE and DA neurons. Although this selectivity is a property shared by other psychotropic drugs for other receptors and other regions, why it occurs is still not well understood. ${ }^{7}$

\section{References}

1. Georgiadis JR, Kringelbach ML. The human sexual response cycle: brain imaging evidence linking sex to other pleasures. Prog Neurobiol. 2012; 98(1): 49-81.

2. Stahl SM. Illustrating the circuits of sexual desire. J Clin Psychiatry. 2010; 71(9): 1113-1114.

3. Stahl SM. Circuits of sexual desire in hypoactive sexual desire disorder. J Clin Psychiatry. 2010; 71(5): 518-519.

4. Pfaus JG. Pathways of sexual desire. J Sex Med. 2009; 6(6): 1506-1533.

5. Woodard TL, Nowak NT, Balon R, Tancer M, Diamond MP. Brain activation patterns in women with acquired hypoactive sexual desire disorder and women with normal sexual function: a cross sectional pilot study. Fertil Steril. 2013; 100(4): 1068-1076.

6. Arnow BA, Millheiser L, Garrett A, et al. Women with hypoactive sexual desire disorder compared to normal females: a functional magnetic resonance imaging study. Neuroscience. 2009; 158(2): 484-502. 


\section{BRAINSTORMS—Clinical Neuroscience Update}

7. Stahl SM, Sommer B, Allers KA. Multifunctional pharmacology of flibanserin: possible mechanism of therapeutic action in hypoactive sexual desire disorder. J Sex Med. 2011; 8(1): 15-27.

8. Stahl SM. Targeting circuits of sexual desire as a treatment strategy for hypoactive sexual desire disorder. J Clin Psychiatry. 2010; 71(7): 821-822.

9. Gelez H, Greggain-Mohr J, Pfaus JG, Allers KA, Giuliano F. Flibanserin treatment increases appetitive sexual motivation in the female rat. J Sex Med. 2013; 10(5): 1231-1239.

10. Gelez H, Clement $\mathrm{P}$, Compagnie S, et al. Brain neuronal activation induced by flibanserin treatment in female rats. Psychopharmacology (Berl). 2013; 230(4): 639-652.

11. Aubert Y, Gustison ML, Gardner LA, et al. Flibanserin and 8-OHDPAT implicate serotonin in association between female marmoset monkey sexual behavior and changes in pair-bond quality. J Sex Med. 2012; 9(3): 694-707.

12. Stahl SM. The last Diagnostic and Statistical Manual (DSM) replacing our symptom-based diagnoses with a brain circuit-based classification of mental illnesses. CNS Spectr. 2013; 18(2): 65-68.

13. Insel $\mathrm{T}$, Cuthbert $\mathrm{B}$, Garvey $\mathrm{M}$, et al. Research Domain Criteria (RDoC): toward a new classification framework for research on mental disorders. Am J Psychiatry. 2010; 167(7): 748-751.

14. Goto Y, Grace AA. Dopaminergic modulation of limbic and cortical drive of nucleus accumbens in goal-directed behavior. Nat Neurosci. 2005; 8: 805-813.

15. American Psychiatric Association. Diagnostic and Statistical Manual of Mental Disorders. 5th ed. Arlington, VA: American Psychiatric Association; 2013.

16. Clayton AH, Goldfischer E, Goldstein I, et al. Validity of the decreased sexual desire screener for diagnosing hypoactive sexual desire disorder. J Sex Marital Ther. 2013; 39(2): 132-143.
17. Stahl SM. The psychopharmacology of sex, Part 1: neurotransmitters and the 3 phases of the human sexual response. J Clin Psychiatry. 2001; 62(2): 80-81.

18. Stahl SM. The psychopharmacology of sex, Part 2: effects of drugs and disease on the 3 phases of human sexual response. J Clin Psychiatry. 2001; 62(3): 147-148.

19. Jayne C, Simon JA, Taylor LV, Kimura T, Lesko LM. Open label extension study of flibanserin in women with hypoactive sexual desire disorder. J Sex Med. 2012; 9(12): 3180-3188.

20. DeRogatis LR, Komer L, Katz M, et al. Treatment of hypoactive sexual desire disorder in premenopausal women: efficacy of flibanserin in the VIOLET study. J Sex Med. 2012; 9(4): 1074-1085.

21. Thorp J, Simon J, Dattani D, et al. Treatment of hypoactive sexual desire disorder in premenopausal women: efficacy of flibanserin in the DAISY study. J Sex Med. 2012; 9(3): 793-804.

22. Goldfischer ER, Breaux J, Katz M, et al. Continued efficacy and safety of flibanserin in premenopausal women with hypoactive sexual desire disorder (HSDD): results from a randomized withdrawal trial. J Sex Med. 2011; 8(11): 3160-3172.

23. Simon JA, Kingsberg SA, Shumel B, Hanes V, Garcia M, Sand M. Efficacy and safety of flibanserin in postmenopausal women with hypoactive sexual desire disorder: results of the SNOWDROP trial. Menopause. 2014; 21(6): 633-640.

24. Katz M, DeRogatis LR, Ackerman R, et al. Efficacy of flibanserin in women with hypoactive sexual desire disorder: results from the BEGONIA trial. J Sex Med. 2013; 10(7): 1807-1815.

25. Bortolozzi A, Masana M, Díaz-Mataix L, et al. Dopamine release induced by atypical antipsychotics in prefrontal cortex requires $5 \mathrm{HT}(1 \mathrm{~A})$ receptors but not $5 \mathrm{HT}(2 \mathrm{~A})$ receptors. Int J Neuropsychopharmacol. 2010; 13(10): 1299-1314. 